\title{
THE USE OF SEPARATED REFLECTION COMPONENTS IN ESTIMATING GEOMETRICAL PARAMETERS OF CURVED SURFACE ELEMENTS
}

\author{
J.C. Glas, F. van der Heijden \\ University of Twente*, The Netherlands
}

\begin{abstract}
Iterative least-squares estimation requires accurate reflectance models to retrieve geometrical parameters of curved surface elements from an image projection. We investigate the use of separating the diffuse (body) reflection from the specular (surface) reflection being responsible for image highlights. Experiments show that the (smooth) diffuse component yields the best convergence properties, while the (sharp) specular component can contribute to the improvement of the noise insensitivity.

Key words: shape from shading, reflectance models, param. eter estimation, reflection component separation.
\end{abstract}

\section{INTRODUCTION}

Retrieving geometrical parameters of the surface elements in a 3-D scene from a 2-D image projection is a non-linear inverse problem for which iterative least-squares estimation can provide an optimal solution when assuming additive Gaussian measurement noise. The method requires a priori knowledge about light, material and camera properties applied in accurate imaging and reflectance models. Korsten et al [1] and De Graaf et al [2] have both demonstrated that such a model based approach to image understanding yields good results in estimating parameters of 3-D objects.

The reflectance from a surface can be modelled as a linear combination of diffuse (body) reflection and specular (surface) reflection. The latter model accounts for the the occurrence upon curved surfaces of the shiny spots called highlights, which we consider to be useful clues for shape from shading rather than inconvenient image disturbances. Yet the convergence properties of an iterative estimator appear to suffer under the sharp reflectance profile of the specular component when taking direct measurements from a highlighted image. We present experiments showing that separation of reflection components may solve this convergence problem and improves the noise insensitivity as well.

\section{Reflection component separation}

Inherent differences in spectral distribution and/or polarization of diffuse and specular reflectance offers possibilities to separate these components in images that contain highlights. The spectral density of

* Department of Electrical Engineering, Control, Systems and Computer Engineering Group, Laboratory for Measurement Science and Instrumentation, P.O. Box 217, $7500 \mathrm{AE}$ Enschede, The Netherlands, E-mail: jaap@mi.el.utwente.nl

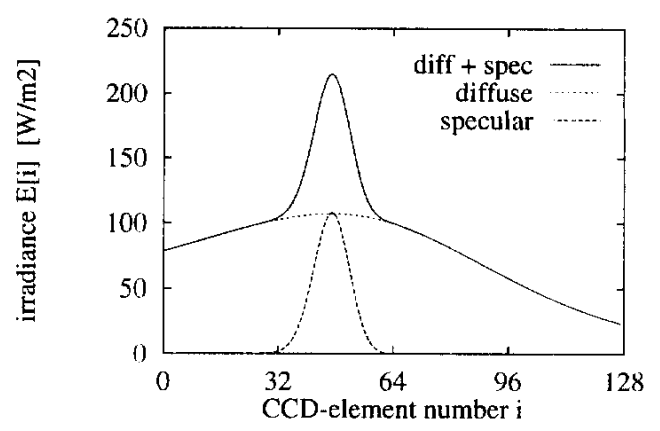

FIGURE 1: Reflection component separation of linescan CCD-camera output without noise.

the diffuse component consists of the product of the spectral densities of the light source and the surface reflectance, while the spectral density of the specular component originates from the light source only: The colour histogramming techniques of Gershon [3] and Klinker [4] utilize this distinction to separate reflection components in colour images. Wolff and Boult [5] demonstrated how the unpolarized diffuse reflectance can be separated from the partially polarized specular reflectance using a rotatable polarization filter in front of the camera. Nayar et al [6] have integrated both separation methods.

Our paper tentatively assumes that the separation between the diffuse and specular reflection components can be established. Figure 1 shows the result of this operation on the output of a line-scan camera. We concentrate on the use of the separated reflection components to the convergence properties and the noise insensitivity of the estimation of geometrical parameters of curved surface elements, upon which highlights will occur frequently.

Based on the image irradiance profiles of the reflection components, one may impute better convergence properties to the smooth diffuse component than to the sharp specular component, because a small change in parameter values will cause a much larger deviation in the measurement of the latter component. This sharpness however is also responsible for a smaller sensitivity to measurement noise, because a large deviation in the measurement will cause only a small deviation in the parameter values. Therefore, the specular component may be utilized in the final stage of the estimation to improve the accurary of especially those parameters that are related to the viewing angle, on which the speru- 


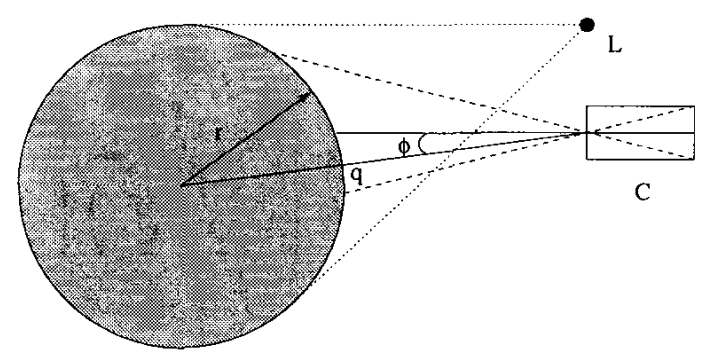

FIGURE 2: Projection of a scene on the $x y$-plane.

lar irradiance unlike the diffuse irradiance depends. We have investigated these hypotheses experimentally and analytically from imaging and reflectance models of a cylindrical world.

\section{MODELLING}

Our world can be conceived of being built from a set of curved surface elements. Currently this primitive has simply been modelled as (part of) a cylinder surface, but our experimental results are extendible to more general parametric surfaces. The part of the world called scene will consist of one solid opaque cylinder aligned with the $z$-axis. Figure 2 shows a top view of such a scene.

The cylinder is irradiated by a single isotropic point light source $L$. The imaging model supplies a perspective projection of the reflectance onto the CCDelements of a pinhole line-scan camera $C$ aligned with the $x y$-plane (see e.g. Van der Heijden [7]). Figure 1 shows an example of the camera output without measurement noise. The occurrence of both a significant diffuse and off-specular reflection component requires the cylinder to be made from a non-conducting nonhomogeneous material such as a plastic.

\section{Reflectance models}

Figure 3 shows a polar plot of the diffuse and specular radiant flux $\Phi[W]$ from a surface patch as a function of the angle $\psi$ between unit surface normal $\vec{N}$ and unit vector $\vec{C}$ towards a CCD-element of the camera. The unit vector $\vec{L}$ pointing from the light source is kept fixed. Unit vector $\vec{R}$ points to the prevalent direction of specular reflection making $\vec{N}$ the bisector of $\vec{L}$ and $\vec{R}$ according to Snell's law.

The total radiance $L_{d+s}$ from the surface patch is a sum of diffuse radiance $L_{d}$ and specular radiance $L_{s}$ (see e.g. Nayar et al [8]):

$$
L_{d+s}=L_{d}+L_{s} \quad\left[W / m^{2} s r\right]
$$

The diffuse (body) reflection mainly results from the scattering of incident radiation inside the material. It can be modelled by the well-known Lambertian reflectance model, in which the exitting radiance does not depend on the viewing angle $\psi$, but only on the angle of incidence $\angle(\vec{L}, \vec{N})$ :

$$
L_{d}=\rho_{d}\left(\vec{L}^{T} \vec{N}\right) L_{L} d \omega_{L} \quad\left[W / m^{2} s r\right]
$$

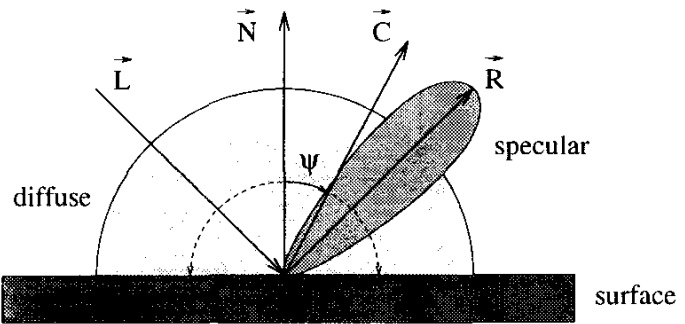

FIGURE 3: Polar plot of diffuse and specular radiant flux $\Phi$ as function of camera angle $\psi$.

A constant diffuse albedo $\rho_{d}$ is supposed. The radiance $L_{L}[W / s r]$ and solid angle $d \omega_{L}[s r]$ of the light source determine the irradiance of the surface. The specular (surface) reflection results from incident radiation directly reflected by the planar microfacets of the surface. In this case the exitting radiance does also depend on the viewing angle $\psi$. If the wavelength of the radiation is considerably smaller than the size of the micro-facets, the geometrical reflectance model of Torrance and Sparrow [9] for the off-specular reflection of roughened surfaces can be used:

$$
L_{s}=\rho_{s} \frac{\mathcal{D}(\vec{C}, \vec{R})}{\vec{C}^{T} \vec{N}} L_{L} d \omega_{L} \quad\left[W / m^{2} s r\right]
$$

The simplification in Eq. (3) applies if the incidence and viewing angles are not extremely oblique. The specular albedo $\rho_{d}$ is supposed to be constant. Assuming that the angle between surface normal $\vec{N}$ and the normal vectors of the planar micro-facets has a Gaussian distribution, the specular reflectance at angle $\beta=\angle(\vec{C}, \vec{R})$ is proportional to the Gaussian density $\mathcal{D}(\beta)$ :

$$
\mathcal{D}(\beta)=\frac{1}{\sigma_{\beta} \sqrt{2 \pi}} e^{-\frac{\beta^{2}}{2 \pi_{\beta}^{2}}}
$$

The surface roughness is determined by the standard deviation $\sigma_{\beta}$.

\section{PARAMETER ESTIMATION}

The parameter estimation of 3-D elements from a 2 -D image projection can be performed by a general method to determine an optimal estimate of an unknown parameter vector $\vec{\alpha}$ from a measurement vector $\vec{\theta}$ given the forward model $\vec{\theta}(\vec{\alpha})$ of the non-linear relationship between them:

$$
\vec{\theta}=\vec{\theta}(\vec{\alpha})+\underline{\vec{n}}
$$

The notation has been adopted from Korsten [1]. In case the additive measurement noise $\underline{\vec{n}}$ has a zeromean Gaussian distribution and a priori knowledge about $\vec{\alpha}$ is unavailable, the optimal estimate of $\vec{\alpha}$ results from minimization of the weighted least-squares error $\ell(\vec{\alpha})$ of the measurement vector:

$$
\ell(\vec{\alpha})=(\vec{\theta}-\vec{\theta}(\vec{\alpha}))^{T} C_{\vec{\theta}}^{-1}(\vec{\theta}-\vec{\theta}(\vec{\alpha}))
$$




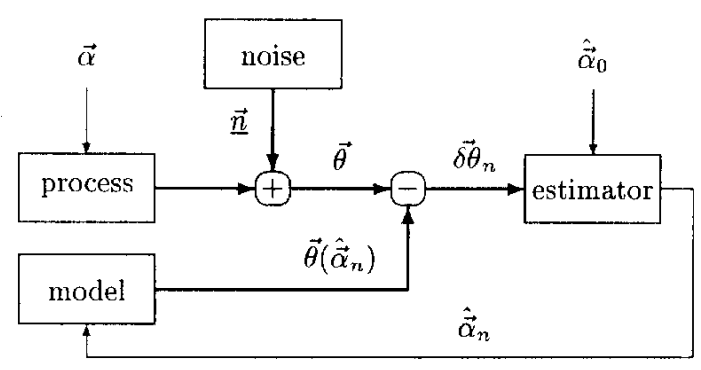

FIGURE 4: Iterative parameter estimation scheme.

The weighting matrix is made by inversion of the measurement covariance matrix $C_{\vec{\theta}}$, which equals the noise covariance $C_{\vec{n}}$.

The non-linearity of $\vec{\theta}(\vec{\alpha})$ makes it difficult to find a straightforward solution for the minimization of Eq. (6). However, if $\vec{\theta}(\vec{\alpha})$ is piecewise differentiable, the Gauss-Newton method can be applied to solve this inverse problem by iterative linearization of $\vec{\theta}(\vec{\alpha})$ around the previous estimate $\hat{\vec{\alpha}}_{n}$ of parameter vector $\vec{\alpha}$. Substituting $\vec{\theta}(\vec{\alpha})$ in Eq. (5) by its first order Taylor approximation around $\hat{\vec{\alpha}}_{n}$ gives:

$$
\vec{\theta}=\left[\vec{\theta}\left(\hat{\vec{\alpha}}_{n}\right)+\left.\frac{\partial \vec{\theta}(\vec{\alpha})}{\partial \vec{\alpha}}\right|_{\vec{\alpha}=\hat{\vec{\alpha}}_{n}}\left(\vec{\alpha}-\hat{\vec{\alpha}}_{n}\right)+\mathcal{R}_{n}\right]+\underline{\vec{n}}
$$

The higher order terms collected in Lagrange's residlual $\mathcal{R}_{n}$ will be considered negligible. Introducing the error vectors $\overrightarrow{\delta \alpha}_{n}$ and $\overrightarrow{\delta \theta}_{n}$ and Jacobian matrix $B_{n}$,

$$
\begin{gathered}
\vec{\delta}_{n}=\vec{\alpha}-\hat{\vec{\alpha}}_{n} \quad \overrightarrow{\delta \theta} \hat{\theta}_{n}=\vec{\theta}-\vec{\theta}\left(\hat{\vec{\alpha}}_{n}\right) \\
B_{n}=\left.\frac{\partial \vec{\theta}(\vec{\alpha})}{\partial \vec{\alpha}}\right|_{\vec{\alpha}=\hat{\vec{\alpha}}_{n}}
\end{gathered}
$$

Eq. (7) can be rewritten into the linearized matrix equation:

$$
\overrightarrow{\delta \theta}_{n}=B_{n} \overrightarrow{\delta \alpha_{n}}+\underline{\vec{n}}
$$

The possibly complex analytical derivation of the Jacobian can be avoided by numerical approximation of matrix $B_{n}$ using finite differences [2].

If the a priori uncertainty about parameter vector $\vec{\alpha}$ is expressed by an infinite covariance $C_{\vec{\alpha}}=\infty I$, Liebelt [10] derives the following unbiased weighted least-squares estimator for $\overrightarrow{\delta \alpha_{n}}$ from Eq (10):

$$
\hat{\vec{\delta} \alpha_{n}}=\left(B_{n}^{T} C_{\vec{\theta}}^{-1} B_{n}\right)^{-1} B_{n}^{T} C_{\vec{\theta}}^{-1} \overrightarrow{\delta \theta} n
$$

In case of white measurement noise, matrix $C_{\vec{\theta}}^{-1}$ is diagonal and can be dropped. The a posteriori covariance matrix $C_{\delta \vec{\alpha}_{n}}$ which equals the parameter covariance $C_{\vec{\alpha}_{n}}$ is given by:

$$
C_{\delta \vec{\alpha}_{n}}=\left(B_{n}^{T} C_{\overrightarrow{\boldsymbol{\theta}}}^{-1} B_{n}\right)^{-1}
$$

The iterative application of Eq. (11) yields the GaussNewton method:

$$
\hat{\vec{\alpha}}_{n+1}=\hat{\vec{\alpha}}_{n}+\hat{\vec{\alpha}}_{n}
$$

\begin{tabular}{|c||c|c|c|}
\hline$\vec{\theta}$ & $=$ & $\operatorname{dim} \vec{\theta}$ & $C_{\vec{\theta}}$ \\
\hline$\vec{\theta}_{d+s}$ & $\vec{E}_{d+s}$ & 128 & $\left(\sigma_{d}^{2}+\sigma_{s}^{2}\right) I$ \\
$\vec{\theta}_{d}$ & $\vec{E}_{d}$ & 128 & $\sigma_{d}^{2} I$ \\
$\vec{\theta}_{s}$ & $\vec{E}_{s}$ & 128 & $\sigma_{s}^{2} I$ \\
$\vec{\theta}_{d \mid s s}$ & $\left(\begin{array}{cc}\vec{E}_{d} \\
\vec{E}_{s}\end{array}\right)$ & 256 & $\left(\begin{array}{cc}\sigma_{d}^{2} I & O \\
O & \sigma_{s}^{2} I\end{array}\right)$ \\
\hline
\end{tabular}

TABLE 1: Measurement vectors $\vec{\theta}$ and their covariance matrices $C_{\vec{\theta}}$.

Figure 4 shows a schematic overview. The algorithm terminates if the decrease in the least-squares error $\ell(\vec{\alpha})$ becomes very small:

$$
\ell\left(\hat{\vec{\alpha}}_{n}\right)-\ell\left(\hat{\vec{\alpha}}_{n+1}\right)<\epsilon
$$

The measure of non-linearity of model $\vec{\theta}(\vec{\alpha})$ and the ability to choose initial estimate $\hat{\vec{\alpha}}_{0}$ close to the real parameter vector $\vec{\alpha}$ determines whether the GaussNewton iteration will converge to a (not necessarily global) minimum of the least-squares error function $\ell(\vec{\alpha})$.

\section{EXPERIMENTAL SET-UP}

All our experiments refer to the simultaneous estimation of the geometrical parameters from the scene of Figure 2. The three-dimensional parameter vector $\vec{\alpha}$ consists of the radius $r$ of the cylinder, its distance $q$ to the camera and its angle $\phi$ with the optical axis. Because the positive quantities radius and distance are not two-sided unlimited, the parameters $q$ and $r$ have been (hosen logarithmic to improve the reliabiliy of the estimator (see Tarantola [11]). All other parameters in the imaging and reflectance models are assumed to be knowri.

Although the behaviour of a non-linear estimator is varying in its parameter space, relevance is preserved when confining the analysis to a representative point in that space. Therefore, our measurement vector $\vec{\theta}$ will always originate from the irradiance profiles of Figure 1 that is considered to be a representative image of a lighlight on the cylinder surface.

\section{Measurement models}

The use of separated reflection components has been studied by applying a number of four different measurement models $\vec{\theta}(\vec{\alpha})$ in the parameter estimation process. Table 1 lists the measurement vectors produced by these models in conformity with Eq. (5). The irradiance of the reflection components is vectorized according to $(\vec{E} \ldots)_{i}=E_{\ldots}[i]$ from Figure 1 . Measurement vector $\vec{\theta}_{d+s}$ contains the unseparated irradiance, $\vec{\theta}_{d}$ the diffuse component, $\vec{\theta}_{s}$ the specular component and the double-sized vector $\vec{\theta}_{d \|_{s}}$ both reflection components in parallel.

The model approximations $\vec{\theta}\left(\hat{\vec{\alpha}}_{n}\right)$ of the measurement vector are generated by a ray-tracer. The lack 

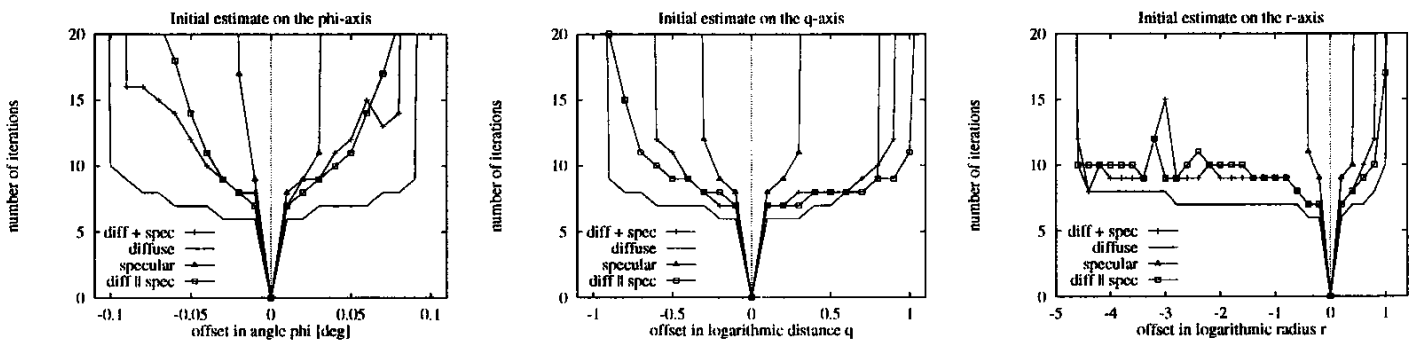

FIGURE 5: Convergence behaviour along the axis of parameter space.

of a physical scene implies that the process generating the real $\vec{\theta}$ has to be simulated by ray-tracing $\vec{\theta}(\vec{\alpha})$ and adding noise $\underline{\vec{n}}$. The measurement vectors $\vec{\theta}_{d}$ and $\vec{\theta}_{s}$ are considered to be affected by additive zero-mean Gaussian noise with no spatial correlation and with constant standard deviations $\sigma_{d}$ and $\sigma_{s}$ respectively. Thermal and fixed pattern noise in CCD-elements satisfies these properties [7]. If the separation in reflection components is established by means of multiple colour or polarization channels, it may be assumed that no correlation exists between the noise in $\vec{\theta}_{d}$ and $\vec{\theta}_{s}$. This leads to the definition of the covariance matrices $C_{\vec{\theta}}$ as listed in Table 1 .

\section{CONVERGENCE MEASUREMENTS}

The measure of non-linearity of the estimation problem mainly determines the convergence speed and area of the estimator. These get worse as neglecting Lagrange's residue $\mathcal{R}_{n}$ in Eq. (7) becomes less justifiable. The influence of noise becomes less significant in that case, which implies that convergence can be measured without adding noise $\left(\sigma_{d}=\sigma_{s}=0\right)$. The choice of initial estimate $\hat{\vec{\alpha}}_{0}$ has been restricted to points on the axes of parameter space, but nevertheless a good impression of the convergence behaviour has been obtained.

Figure 5 shows the number of iterations required to converge as function of the distance between initial estimate and real solution. The straight vertical lines indicate divergence. The diffuse component $\vec{\theta}_{d}$ gives the best convergence properties. Its convergence speed especially prevails at initial deviations in angle $\phi$, because the sharp specular component causes large errors $\ell(\vec{\alpha})$ in that case. In some cases measurement model $\vec{\theta}_{d \|_{s}}(\vec{\alpha})$ provides a somewhat larger convergence area which indicates that specularities may be helpful in the future to obtain close initial estimates.

\section{NOISE SENSITIVITY ANALYSIS}

The noise sensitivity of a model is determined by the covariance matrix $C_{\vec{\alpha}_{\infty}}$ of a mean final estimate $\hat{\vec{\alpha}}_{\infty}$ given a fixed measurement covariance $C_{\vec{\theta}}$. Instead of estimating $\hat{\vec{\alpha}}_{\infty}$ for a whole set of noise realizations, we only have to consider $\underline{\vec{n}}=\overrightarrow{0}$. This realization converges to the real parameter vector $\vec{\alpha}$ which is the mean $\hat{\vec{\alpha}}_{\infty}$ for an unbiased estimator. The corresponding covariance matrix $C_{\vec{\alpha}_{\infty}}=C_{\vec{\delta}_{\infty}}$ follows from Eq. (12):

$$
C_{\delta \vec{\alpha}_{\infty}}=\left(B_{\infty}^{T} C_{\vec{\theta}}^{-1} B_{\infty}\right)^{-1}
$$

If the error vector $\overrightarrow{\delta \alpha_{n}}$ has a Gaussian distribution, the probability density is defined by the following quadratic form (see Sorensen [12]):

$$
\overrightarrow{\delta \alpha}_{\infty}^{T} C_{\delta \alpha_{\infty}}^{-1} \overrightarrow{\delta \alpha_{\infty}}=c^{2}
$$

Because $C_{\overrightarrow{\delta \alpha_{\infty}}}^{-1}$ is positive definite, the equation decribes an error ellipsoid $\mathcal{E}$ in parameter space around the final estimate $\hat{\vec{\alpha}}_{\infty}$. This surface of constant probability bounds the $c-\sigma$ confidence interval.

Figure 6 shows principle plane intersections through the centre $\hat{\vec{\alpha}}_{\infty}$ of the error ellipsoids for fixed $\sigma_{d}=\sigma_{s}$. These intersections correspond with the error ellipses of estimation problems in which two parameters of $\vec{\alpha}$ are unknown and the third one is known. The 3-D impression of only ellipsoids $\mathcal{E}_{d}$ and $\mathcal{E}_{s}$ can be found in Figure 7. These ellipsoids scale with the value of $\sigma_{d}$ and $\sigma_{s}$ respectively. The ellipsoid $\mathcal{E}_{d \| s}$ is always enclosed by both the ellipsoids $\mathcal{E}_{d}$ and $\mathcal{E}_{s}$. The influence of increasing noise in one reflection component on the sensitivity of measurement model $\vec{\theta}_{d \| s}(\vec{\alpha})$ will be bounded by the other reflection component. This principle does not apply for the unseparated measurement model $\vec{\theta}_{d+s}(\alpha)$, because ellipsoid $\mathcal{E}_{d+s}$ scales with $\sqrt{\sigma_{d}^{2}+\sigma_{s}^{2}}$. Again the enclosure of the (aligning) ellipsoid $\mathcal{E}_{d \| s}$ is guaranteed so that model $\vec{\theta}_{d \| s}(\vec{\alpha})$ will always yield the best noise insensitivity. Its double amount of measurements makes the difference with $\vec{\theta}_{d+s}(\vec{\alpha})$ in case $\sigma_{d}=\sigma_{s}$.

The first two intersections of Figure 6 indeed show that the specular component $\vec{\theta}_{s}$ significantly contributes to the the accuracy of angle $\phi$ for $\sigma_{d}=\sigma_{s}$. This improvement however depends on the $\sigma_{d}$ to $\sigma_{s}$ ratio of the reflection components or actually on their signal to noise ratios when considering highlighted images in general. The third intersection shows that separating reflection components has not solved the size-depth $(r-q)$ problem of monocular vision. 

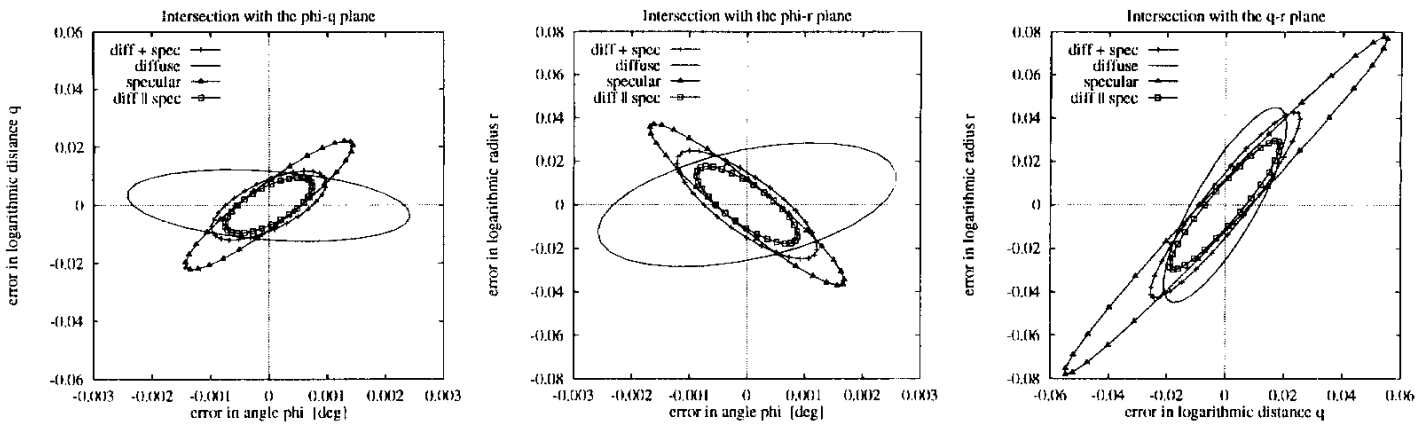

FIGURE 6: Principle plane intersections of the 1- $\sigma$ error ellipsoids for $\sigma_{d}=\sigma_{s}$.

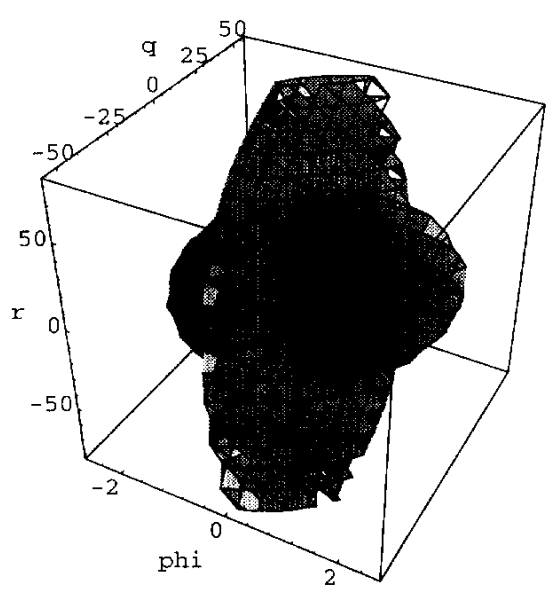

FIGURE 7: A 3-D impression of the (dark) diffuse and (light) specular 1- $\sigma$ error ellipsoids.

\section{CONCLUSIONS}

The main conclusion is that separated reflection components benefit the estimation of geometrical parameters of curved surface elements. The best strategy in terms of convergence properties and noise insensitivity starts estimating with the smooth diffuse component only to attain fast convergence. Modelling also the sharp specular reflection appears to be useful in the ultimate stage of the estimation. A changeover to a measurement vector containing the diffuse and specular components in parallel will improve the insensitivity of the final estimate to measurement noise.

\section{Discussion}

Uncorrelated additive Gaussian measurement noise has been assumed throughout this paper. Only a worse separation method results in reflection components with anti-correlated noise that would weaken our conclusions about noise insensitivity. Quantum noise has a Poisson distribution and fluctuations with respect to the reflectance model cause multiplicative noise [7]. Although a least-squares estimator is not optimal for signal-dependent noise, experiments with multiplicative noise indicate that the principles of reflection conıponent separation still apply. In the first place however, images from a real scene are required to define a realistic noise model.

\section{REFERENCES}

[1] Korsten M.J., 1989, "Three-Dimensional Body Parameter Estimation from Digital Images", Ph.D. Thesis, University of Twente, The Netherlands.

[2] De Graal A.J., Korsten M.J., Houkes Z., 1990, "Estimation of fosition and Orientation of Objects from Stereo Images", Proc. 12. DAGM-Symposium, Mustererkennung 1990, Informatik-Fachberichte 254, OberkochenAalen, Giermany, 348-355.

[3] Gershon R., 1987, "The Use of Color in Computational Vision", Ph.D. Thesis, University of Toronto, Canada.

[4] Klinker (4.J., 1988, "A Physical Approach to Color Image Understanding", Ph.D. Thesis, Carnegie Mellon University, USA

[5] Wolff L.13., Boult T.E., 1991, "Constraining Object Features Using a Polarization Reflection Model", IEEE Tr. Patt. Arial. Mach. Intell., 13-7, 635-657.

[6] Nayar S.K., Fang X.S., Boult T., 1993, "Removal of Specularities Using Color and Polarization", Proc. IEEE Comp. Vis. Patt. Recogn., New York, USA, 583-590.

[7] Van der Heijden F., 1994, "Image Based Measurement Systems. Object Recognition and Parameter Estimation", John Wiley \& Sons, Chicester, UK.

[8] Nayar S K., Ikeuchi K., Kanade T., 1991, "Surface Reflection: Physical and Geometrical Perspectives", IEEE Tr. Patt. Anal. Mach. Intell, 13-7, 611-634.

[9] Torrancu K., Sparrow E., 1967, "Theory for Off-Specular Reflection from Roughened Surfaces", J. Opt. Soc Am $A, 57-7,1105-1114$.

[10] Liebeit P.B., 1967, "An Introduction to Optimal Estimation". Addison-Wesley Publishing Company, Reading, USA.

[11] Tarantola A., 1987. "Inverse Problem Theory, Methods for I lata Fitting and Model Parameter Estimation", Elsevier. Amsterdam, The Netherlands

[12] Sorensen H.W., 1980, "Parameter Estimation, Principles and Problems", Control and Systems Theory, Vol. 9, Marcel Jekker, New York, USA 